\title{
Quantitative analysis of the anterolateral ossification mass in diffuse idiopathic skeletal hyperostosis of the thoracic spine
}

\author{
J. J. Verlaan · L. A. Westerveld · J. W. van Keulen • \\ R. L. A. W. Bleys $\cdot$ W. J. Dhert $\cdot$ J. A. van Herwaarden • \\ F. L. Moll $\cdot$ F. C. Oner
}

Received: 10 February 2010/Revised: 20 January 2011/Accepted: 6 February 2011/Published online: 22 February 2011

(C) The Author(s) 2011. This article is published with open access at Springerlink.com

\begin{abstract}
Diffuse idiopathic skeletal hyperostosis (DISH) is a systemic condition leading to ossification of spinal ligaments and has been shown to behave similarly to ankylosing spondylitis (AS) often leading to unstable hyperextension fractures. Currently, no quantitative data are available on the spatial relationship between the bridging anterolateral ossification mass (ALOM) and the vertebral body/intervertebral disc to explain the propensity in DISH to fracture through the vertebral body instead of through the intervertebral disc as more often seen in AS. Furthermore, no reasonable explanation is available for the typical flowing wax morphology observed in DISH. In the current study, a quantitative analysis of computed tomography (CT) data from human cadaveric specimens with DISH was performed to better understand the newly formed osseous structures and fracture biomechanics. Additionally, the results were verified using computed tomography angiography data from ten patients with DISH and ten controls. Transverse CT images were analyzed to obtain ALOM area and centroid angle relative to the anteroposterior axis; intervertebral disc and adjacent cranial and caudal levels. The ALOM area at the midvertebral body level averaged $57.9 \pm 50.0 \mathrm{~mm}^{2}$; at the
\end{abstract}

J. J. Verlaan $(\bowtie)$. L. A. Westerveld · W. J. Dhert · F. C. Oner Department of Orthopedics, University Medical Center Utrecht, Heidelberglaan 100, 3584 CX Utrecht, The Netherlands e-mail: j.j.verlaan@umcutrecht.nl

J. W. van Keulen · J. A. van Herwaarden · F. L. Moll Department of Vascular Surgery, University Medical Center Utrecht, Heidelberglaan 100, 3584 CX Utrecht, The Netherlands

R. L. A. W. Bleys

Department of Anatomy, University Medical Center Utrecht, Heidelberglaan 100, 3584 CX Utrecht, The Netherlands mid-intervertebral disc space level it averaged $246.4 \pm 95.9 \mathrm{~mm}^{2}$. The mean ALOM area at the adjacent level caudal to the mid-vertebral body level was $169.6 \pm 81.3 \mathrm{~mm}^{2}$; at the adjacent cranial level, it was $161.7 \pm 78.2 \mathrm{~mm}^{2}$. The main finding was the significant difference between mean ALOM area at the mid-vertebral body level and other three levels $(p<0.0001)$. The subsequent verification study showed the presence of vertebral segmental arteries at the mid-vertebral body level in nearly all images irrespective of the presence of DISH. A larger area of ALOM seemed associated with increased counterclockwise rotation (away from the aorta) of the centroid relative to the anteroposterior axis. The results from the present study suggest a predisposition for fractures through the vertebral body and a role for the arterial system in the inhibition of soft tissue ossification.

Keywords Diffuse idiopathic skeletal hyperostosis . Ossification - New bone formation - Ankylosed spine . Vertebral segmental artery $\cdot$ Hyperextension fracture

\section{Introduction}

Diffuse idiopathic skeletal hyperostosis (DISH) is a systemic condition leading to ossification of ligaments and entheses $[5,12]$. A hallmark of DISH is the flowing ossification (often compared to flowing candle wax) of anterolateral soft tissue over at least four contiguous vertebral bodies, most frequently found in the thoracic spine [12]. Clinical symptoms resulting from DISH are mainly due to altered biomechanics and may lead to a decreased range of motion and painful stiffness of the structures affected $[6,11]$. Direct compression of surrounding tissues by the progressively growing bony mass in the cervical spine has 
led to several cases of dysphagia, airway obstruction, and radicular complaints [8]. Furthermore, advanced forms of spinal DISH have shown to behave similarly to ankylosing spondylitis (AS), increasing the chance for unstable spine fractures four- to eight-fold compared to a general population, even after low energy trauma [3,9]. In a recent systematic review of the literature on the clinical outcome of patients with fractures of the ankylosed spine, an unusually high percentage of hyperextension type fractures (74.4\% of all fractures in AS and $51.2 \%$ of all fractures in DISH) was found, sharply contrasting the percentage of $0.2 \%$ hyperextension fractures (type B3, according to the AO classification) found by Magerl et al., in their pivotal study of 1,445 spine fractures [7, 14]. Remarkably, the planes of the fractures sustained by patients with DISH were more frequently located through the vertebral body than through the disc (Fig. 1). As the predilection place for fractures in AS seems to be the opposite, the two ankylosing disorders may constitute different biomechanical configurations with distinct modes of failure [1]. Currently, no quantitative data are available on the spatial relationship between the bridging anterolateral bony mass and the vertebral body/intervertebral disc in DISH to explain the different fracture pattern compared to AS. Additionally, no reasonable explanation beyond speculation has been published for the development of the typical, right-sided 'flowing candle wax' morphology, as observed in DISH.

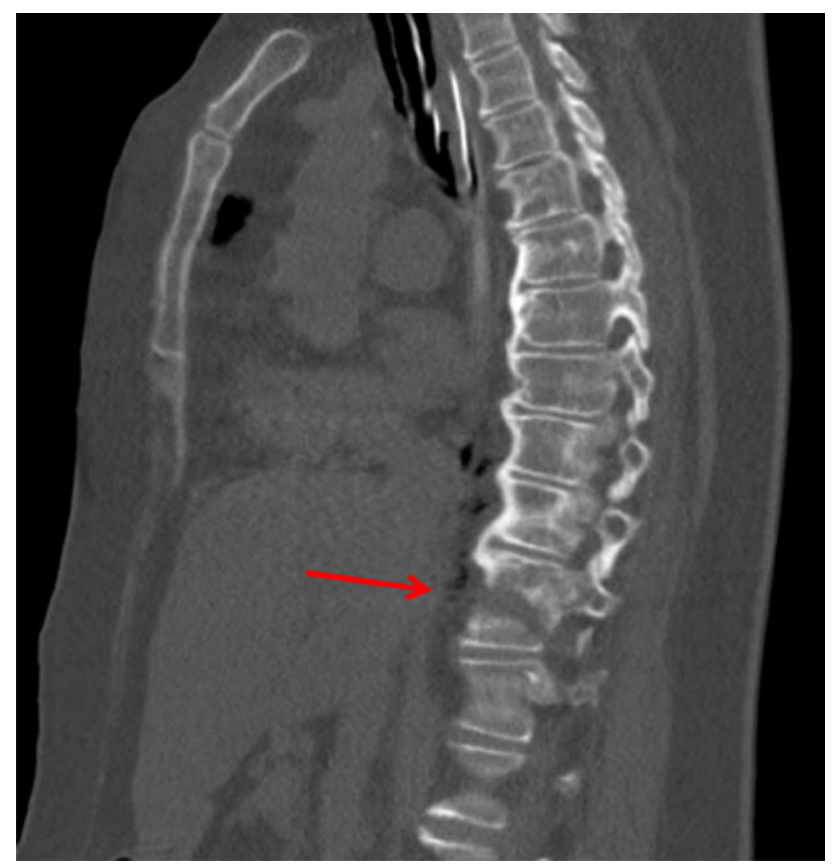

Fig. 1 Sagittal reconstruction of a computed tomography scan of a 68-year-old female pedestrian who was hit from behind by a motor vehicle, demonstrating diffuse idiopathic skeletal hyperostosis at the levels T5-T10 and a T10 hyperextension fracture through the vertebral body (arrow)
The hypotheses of the present study were therefore twofold:

1. the spatial characteristics of newly formed osseous structures in DISH may be responsible for the higher incidence of fractures through the vertebral body rather than through the disc space;

2. the presence of the arterial vascular system may provide a natural barrier for new bone formation in DISH.

To answer the first hypothesis, the extent and localization of DISH were investigated by quantifying the transverse area and centroid (geometric center) of the anterolateral ossified mass on computed tomography (CT) data obtained from ten human cadaveric thoracic spines with DISH. To test the second hypothesis, the results obtained from the first experiment were verified by localizing the vertebral segmental arteries (VSA) on computed tomography angiography (CTA) data of ten patients with DISH and subsequently compared to ten controls.

\section{Materials and methods}

\section{Investigating the extent and localization of DISH}

A total of 89 embalmed human cadaveric thoracic spines, stripped from their surrounding soft tissues, were obtained from the Dept. of Anatomy of our institution and screened for DISH, by two independent observers, by macroscopical inspection and fluoroscopy (OmnidiagnostEleva; Philips Medical Systems, Best, the Netherlands) using the criteria of Resnick et al. [12]. The absence of sacroiliac erosion was not considered an absolute requirement for this part of the study since these joints were not available for screening in the specimens obtained. A total of ten specimens (five male/five female donors, on average 80.4 years old, ranging from 66 to 91 years) met the criteria and underwent CT to:

1. establish a definite diagnosis of DISH (excluding AS and other osseous abnormalities); and

2. perform quantitative analysis of the anterolateral ossifications.

Scanning was performed in supine position with a 64-slice scanner (Philips Brilliance; PhilipsMedical Systems, Best, the Netherlands), using $0.625-\mathrm{cm}$ axial helical scans with high resolution $(120 \mathrm{kV}, 200 \mathrm{mAs}$, slice thickness $0.9 \mathrm{~mm}$ ). On sagittal and coronal reconstructions, the level of interest was determined by identifying and recording the first four contiguous spinal levels connected by an anterolateral ossification mass (ALOM), counting from the twelfth thoracic vertebra and moving cranially. 


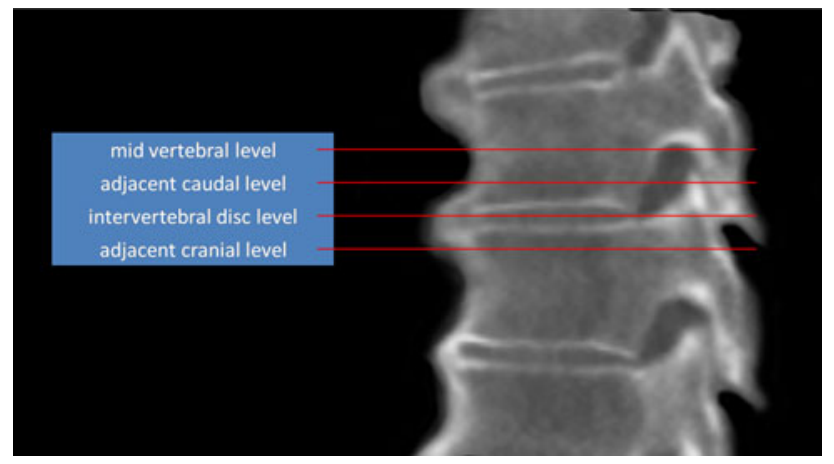

Fig. 2 Sagittal reconstruction of a computed tomography scan of a human cadaveric spine with DISH demonstrating the transverse levels used for quantitative measurements (the description 'cranial' or 'caudal' is relative to the closest mid-vertebral level)

From all four-segment specimens thus identified, the midvertebral body level; intervertebral disc space level and inbetween levels were visualized. See also Fig. 2 for a detailed illustration of the location of the levels under study. A total of $130 \mathrm{CT}$ images (10 specimens $\times 13$ transverse planes) were captured in .bmp format $(768 \times 765$ pixels) and subsequently imported to, and analyzed by, 2D/3D modeling software (Rhinoceros ${ }^{\circledR}$ version 3.0, Seattle, USA). First, the anteroposterior (AP) axis was defined by the line drawn from the most ventral to the most dorsal part of the vertebral body/disc. This AP line was bisected to create a vertebral body center node (VBC). Multiple nodes (up to 50) were subsequently drawn manually to outline the ALOM and a connecting line was drawn through these nodes (see Fig. 3). The centroid node (geometrical center; $\mathrm{CN}$ ) of the ALOM was calculated and automatically drawn by the software and a line was drawn from the $\mathrm{CN}$ to $\mathrm{VBC}$ in order to measure the angle of this line relative to the AP axis, called the anteroposterior- centroid node angle (AP-CN angle). Finally, a line was drawn over the CT-generated ruler to convert to a realworld dimension $(\mathrm{mm})$. Total ALOM area was calculated by the software (in $\mathrm{mm}^{2}$ ) as was the AP-CN angle (in ${ }^{\circ}$, where a positive value was assigned to all orientations pointing to the right side of the body, i.e. counter-clockwise from the AP axis, and a negative value assigned to orientations pointing to the left side of the body, i.e. clockwise from the AP axis).

Localization of vertebral segmental arteries in DISH and controls

The results from the first experiment were verified using computed tomography angiography data (using the same scanner and scanning protocol described above) obtained from 20 patients examined previously for aneurysms of the abdominal aorta, ten of whom also had DISH according to the full set of Resnick criteria (all male, age on average 71.4 years, ranging from 57 to 85 years) while the diagnosis DISH was definitely ruled out for the other ten patients (all male, age on average 68.9 years, ranging from 58 to 80 years) again by two independent observers. The tenth thoracic vertebra, a level frequently involved in DISH and present in the majority of the cadaveric specimens used, was identified on the CTA scans and coronal images were obtained from the following three locations: the anterior vertebral body wall; the center of the vertebral body and the plane in-between (see Fig. 4). On these images the right-sided VSA was identified and its cranialcaudal location was recorded using the same levels as for the cadaveric experiment: at the mid-vertebral body level; intervertebral disc space level and in-between adjacent levels.

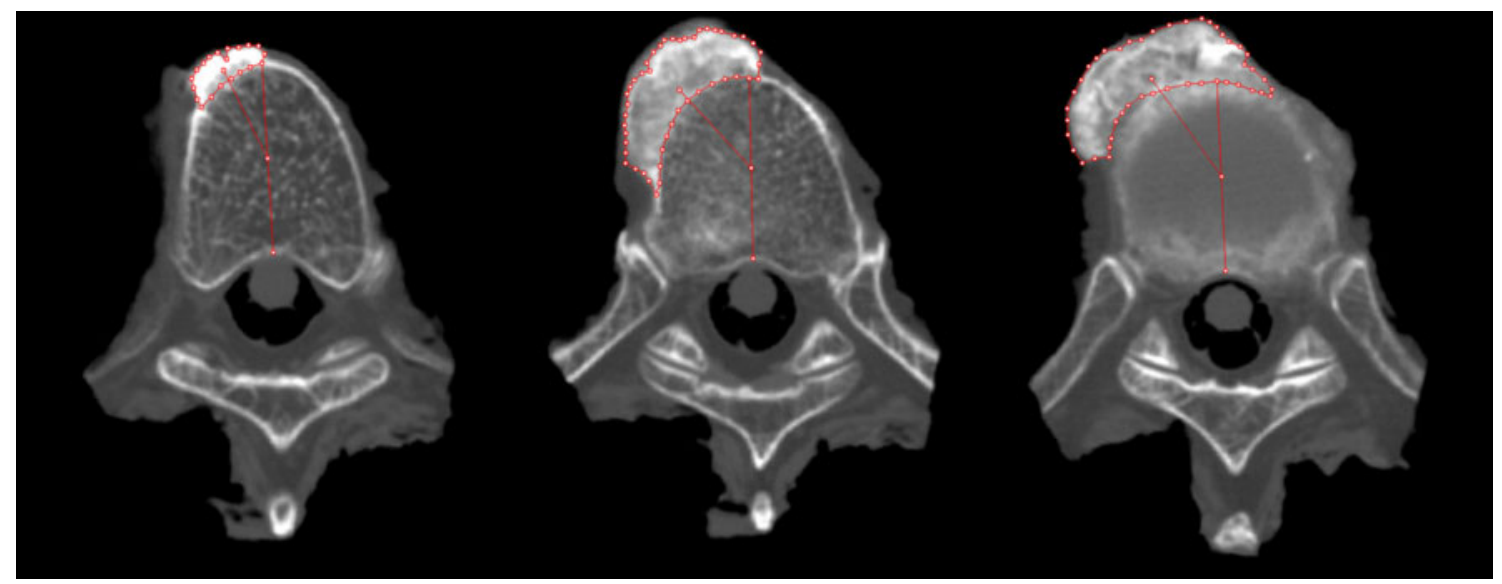

Fig. 3 Three processed transverse computed tomography images from the same cadaveric specimen (left at mid-vertebral level, middle at adjacent cranial level, right at intervertebral disc space level) showing the graphical method used to delineate the anterolateral ossification mass and angle between the centroid and anteroposterior axis 


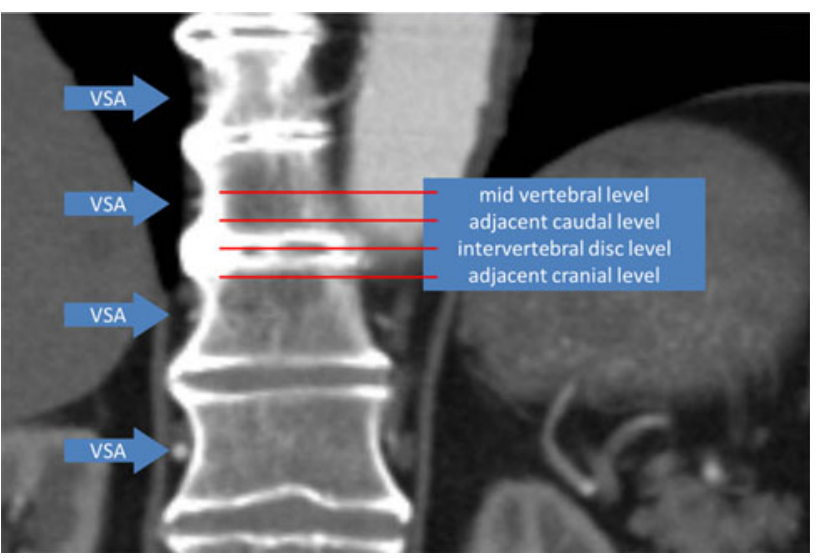

Fig. 4 Coronal reconstruction of a computed tomography angiography scan of a patient with DISH demonstrating the method of locating the vertebral segmental artery (VSA)

\section{Statistics}

A repeated measures analysis with two within factors [affected vertebral level $\left(\mathrm{T}_{n}\right)$ and measurement localization] was used to examine the main effects of these two factors but also to assess whether these effects depend on each other (interaction between vertebral level and measurement localization). If there was a significant main effect, a post hoc analysis with Bonferroni adjustment was performed. For the measurement localization as well as for the vertebral levels, the most cranial location/level respectively, was compared with all others. Statistical significance was set at $p<0.05$.

\section{Results}

Investigating the extent and localization of DISH

ALOM, bridging (at least) four levels, was found at T6-T9 (in three specimens); T7-T10 (four specimens) and T8T11 (three specimens). The ALOM area at the mid-vertebral body level from T6, T7, T8, T9, T10 and T11 averaged $57.9 \pm 50.0 \mathrm{~mm}^{2}$; at the mid-intervertebral disc space level from T6-T7, T7-T8, T8-T9, T9-T10 and T10-T11 it averaged $246.4 \pm 95.9 \mathrm{~mm}^{2}$. The mean ALOM area at the adjacent level caudal to the mid-vertebral body level was $169.6 \pm 81.3 \mathrm{~mm}^{2}$; the mean ALOM area at the adjacent cranial level was $161.7 \pm 78.2 \mathrm{~mm}^{2}$. No interaction was found between vertebral level and measurements $(p=0.19)$ and no main effect was found for vertebral level $(p=0.63)$. However, there was a significant main effect of measurement localization $(p<0.001)$. The results of the several levels were statistically indifferent from each other $(p>0.6)$ but the measurements within these levels were statistically different $(p<0.001)$. The main finding was the

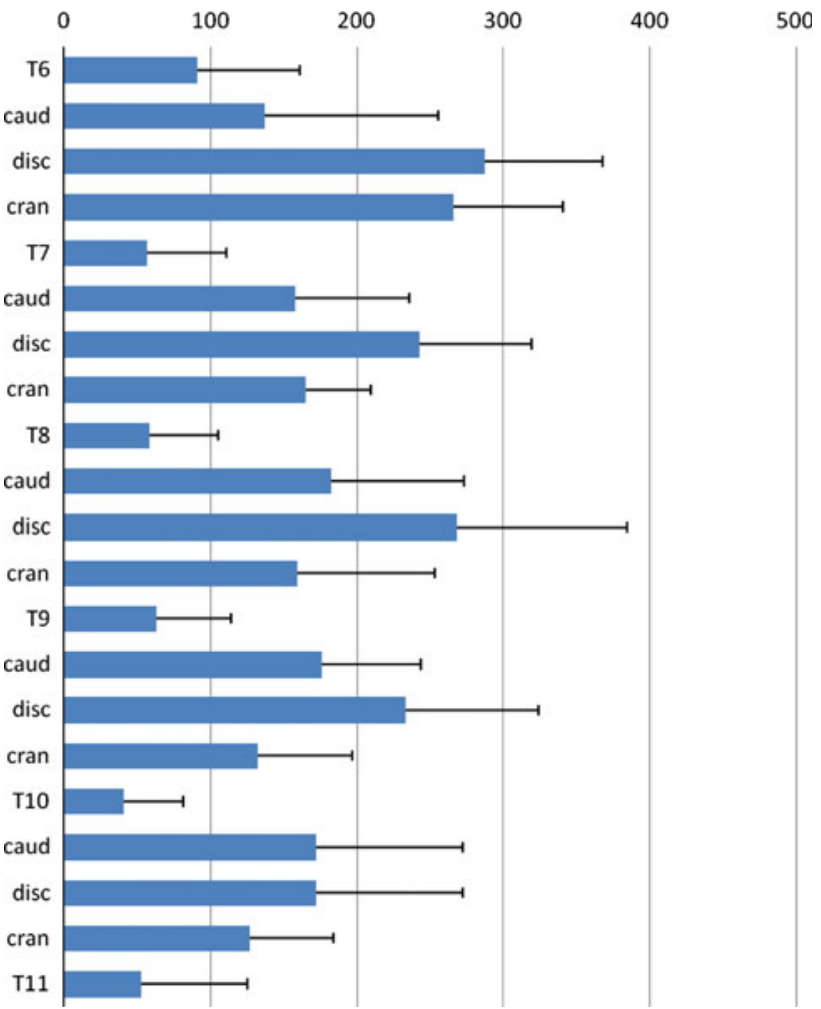

Fig. 5 Graph demonstrating mean area of anterolateral ossification mass values and standard deviations in square millimeters for the individual levels

significant difference between mean ALOM area at the mid-vertebral body level and the other three levels $(p<0.0001)$. See also Fig. 5 for a graphical representation of the results.

The mean AP-CN angle at the mid-vertebral body level was $32.5^{\circ} \pm 25.4^{\circ}$; at the mid-intervertebral disc space level it was $43.7^{\circ} \pm 18.1^{\circ}$. The mean $\mathrm{AP}-\mathrm{CN}$ angle at the adjacent level caudal to the mid-vertebral body level was $41.3^{\circ} \pm 23.3^{\circ}$; at the adjacent level cranial to the midvertebral body level, it was $46.8^{\circ} \pm 22.9^{\circ}$. There was no interaction between level and measurements $(p=0.33)$ and no main effect of vertebral level $(p=0.10)$. A trend was found towards a main effect of the measurement localization, but it was not statistically significant $(p=0.06)$. The results of the several levels were statistically indifferent from each other $(p>0.1)$. The several measurements within these levels were statistically indifferent from each other $(p>0.1)$.

Localization of vertebral segmental arteries in DISH and controls

The VSA could be identified on all 30 images in the DISH group ( $n=10$ cases with three images each) as well as on the 30 images in the control group. In all DISH cases, the 
VSA was located at the mid-vertebral body level or between the mid-vertebral body level and adjacent caudal level. In the control group the VSA was located at the midvertebral body level or between the mid-vertebral body level and adjacent caudal level in 29 images; in one image (located on the anterior vertebral wall) it was at the adjacent caudal level. No significant differences were found between the DISH group and controls regarding the location of the VSA.

In summary, the mean area of ALOM was the largest at the disc space level and smallest at the mid-vertebral body level. The vertebral segmental artery could be located in the coronal plane at, or approximately at, the mid-vertebral body level in nearly all images (59 out of 60) of both DISH cases and controls. A larger area of ALOM was associated with a trend toward increased counter-clockwise rotation of the centroid of this mass relative to the anteroposterior axis.

\section{Discussion}

In the first part of this study, the mean area of the ALOM and its centroid-to-AP axis angle was quantified at the midvertebral body level, mid-intervertebral disc level, and in-between adjacent caudal/cranial levels. It was demonstrated that a significant difference in mean ALOM area exists between these levels, displaying the largest ALOM area at the intervertebral disc level and smallest ALOM area at the mid-vertebral body level. This finding could help explain the previously published high prevalence of (hyperextension-) fractures through the vertebral body in DISH compared to the through-disc fracture pattern more frequently seen in AS [14]. Although little is known about the histology, ultrastructure or yield strength of the ossified soft tissue mass in DISH, it seems plausible that the significantly larger area of ALOM at the intervertebral disc space level and caudal/cranial adjacent levels compared to the mid-vertebral body level could account for the solid bridge between two adjacent endplates leaving the midvertebral level the weakest link. Moreover, a series of anteriorly connected (i.e., ankylosed) vertebral segments could lead to long lever arms on which relatively minor traumatic forces could act to result in hyperextension type fractures (Fig. 1) [4]. The disproportionally large number of hyperextension fractures in DISH relative to compression or flexion fractures (comprising the majority of spinal fractures) is not readily explained by this hypothesis, however.

The counter-clockwise position of the ALOM centroid relative to the AP axis was found to be positively associated with the extent of ALOM area, although this finding did not reach statistical significance. This finding corroborates previously postulated suggestions that the presence of the pulsating aorta prevents DISH from forming [10]. The right-sided localization of the ALOM is highly suggestive for an aortic 'natural barrier', as is the fact that ALOM 'growth' can, apparently, only take place more distant (i.e. counter-clockwise) from this vascular structure. Indeed, previous studies in individuals with situs inversus totalis and DISH, found ALOM formation at the left-side (clockwise) position of the vertebral body [2]. Building further on the hypothesis that only limited amounts of new bone form in the vicinity of a pulsating vascular structure, the results from the cadaveric experiment, showing significantly less new bone formation at the mid-vertebral body level, needed verification by identifying such a structure at that location. The clinical CTA study showed the location of the vertebral segmental artery, indeed, to be situated at (or approximately at) the level of the mid-vertebral body in both DISH cases and controls [13]. This finding strongly suggest the VSA to be a fixed natural barrier for the expansive ossification process possibly dictating the typical 'flowing candle wax' appearance of DISH. It can be argued that CTA data from patients under monitoring for aneurysms of the abdominal aorta might not represent the ideal substance for this study since a preexistent vascular disease could have direct altering effects on the location, orientation, and diameter of branching vertebral segmental arteries. However, to our knowledge, no association between abdominal aneurysms and aberrant vertebral segmental arteries has yet been reported and this potential study limitation has, therefore, not been judged overtly relevant.

Concluding, in DISH, the ossified anterolateral mass area and centroid angle of the ossified anterolateral mass vary considerably at the mid-vertebral; intervertebral and in-between adjacent levels. The results from the present study suggest a predisposition for fractures through the vertebral body and a role for the arterial system in the inhibition of soft tissue ossification in DISH.

Conflict of interest No funding was received in preparation of this work and no conflicts of interest are declared.

Open Access This article is distributed under the terms of the Creative Commons Attribution Noncommercial License which permits any noncommercial use, distribution, and reproduction in any medium, provided the original author(s) and source are credited.

\section{References}

1. Burkus JK, Denis F (1994) Hyperextension injuries of the thoracic spine in diffuse idiopathic skeletal hyperostosis. Report of four cases. J Bone Joint Surg Am 76:237-243

2. Ciocci A (1987) Diffuse idiopathic skeletal hyperostosis (DISH) and situs viscerum inversus. Report of a single case. Clin Exp Rheumatol 5:159-160 
3. de Peretti F, Sane JC, Dran G, Razafindratsiva C, Argenson C (2004) Ankylosed spine fractures with spondylitis or diffuse idiopathic skeletal hyperostosis: diagnosis and complications. Rev Chir Orthop Reparatrice Appar Mot 90:456-465

4. Hendrix RW, Melany M, Miller F, Rogers LF (1994) Fracture of the spine in patients with ankylosis due to diffuse skeletal hyperostosis: clinical and imaging findings. AJR Am J Roentgenol 162:899-904

5. Mader R (2003) Diffuse idiopathic skeletal hyperostosis: a distinct clinical entity. Isr Med Assoc J 5:506-508

6. Mader R, Dubenski N, Lavi I (2005) Morbidity and mortality of hospitalized patients with diffuse idiopathic skeletal hyperostosis. Rheumatol Int 26:132-136

7. Magerl F, Aebi M, Gertzbein SD, Harms J, Nazarian S (1994) A comprehensive classification of thoracic and lumbar injuries. Eur Spine J 3:184-201

8. Oppenlander ME, Orringer DA, La Marca F, McGillicuddy JE, Sullivan SE, Chandler WF, Park P (2009) Dysphagia due to anterior cervical hyperosteophytosis. Surg Neurol 72:266-270 (discussion 270-1)
9. Paley D, Schwartz M, Cooper P, Harris WR, Levine AM (1991) Fractures of the spine in diffuse idiopathic skeletal hyperostosis. Clin Orthop 267:22-32

10. Ravn-Pedersen P, Jurik AG (1988) Diffuse idiopathic skeletal hyperostosis in a patient with right-sided aorta. Rontgenblatter 41:495-496

11. Resnick D, Shaul SR, Robins JM (1975) Diffuse idiopathic skeletal hyperostosis (DISH): Forestier's disease with extraspinal manifestations. Radiology 115:513-524

12. Resnick D, Niwayama G (1976) Radiographic and pathologic features of spinal involvement in diffuse idiopathic skeletal hyperostosis (DISH). Radiology 119:559-568

13. Shimizu S, Tanaka R, Kan S, Suzuki S, Kurata A, Fujii K (2005) Origins of the segmental arteries in the aorta: an anatomic study for selective catheterization with spinal arteriography. AJNR Am J Neuroradiol 26:922-928

14. Westerveld LA, Verlaan JJ, Oner FC (2009) Spinal fractures in patients with ankylosing spinal disorders: a systematic review of the literature on treatment, neurological status and complications. Eur Spine J 18:145-156 